\title{
Improvement of the Rectal Bioavailability of Latamoxef Sodium by Adjuvants Following Administration of a Suppository
}

\author{
Kunio Nakanishi, ${ }^{*, a}$ Tohru Masukawa, ${ }^{a}$ Mikio Masada, ${ }^{b}$ and Tanekazu NAdAI ${ }^{a}$ \\ Faculty of Pharmaceutical Sciences, Setsunan University, ${ }^{a}$ Nagaotoge-cho Hirakata, Osaka 573-01, Japan and \\ Department of Hospital Pharmacy, Fukui Medical School, ${ }^{b}$ Matsuoka, Yoshida-gun, Fukui 910-11, Japan. \\ Received April 25, 1994; accepted July 4, 1994
}

\begin{abstract}
The absorption of an antibiotic, latamoxef sodium (LMOX), following the rectal administration of a suppository containing adjuvants was investigated. A lipophilic base (Witepsol H15) was used. The rectal absorption of LMOX following the administration of a suppository without adjuvants was very low. Diclofenac sodium (DF) was used as an absorption promoter; it enhances rectal membrane permeability. The blood level of LMOX following the addition of DF(10 mg) to the base was increased only about 1.3-fold compared with that achieved with LMOX alone (difference not significant); even with a higher dose of DF, the absorption of LMOX was not sufficient.

The release rate of LMOX from the base was slow. When Tween 80, a non-ionic surfactant, was added to improve the release rate of LMOX, the rate was sufficiently increased. The rectal absorption of LMOX on the addition of both Tween 80 and DF was markedly increased compared to that achieved with LMOX alone or with DF.

These results indicate that the rectal absorption of LMOX after administration by a suppository was sufficiently improved by enhancing both the release rate from the base and the membrane permeability of the rectum. Lymphatic uptake and blood levels of LMOX were also investigated after the rectal administration of the LMOX preparation containing both Tween 80 and DF; the lymphatic uptake of LMOX was significantly enhanced compared with the LMOX preparation in which only DF was used as an adjuvant. The mechanism whereby adjuvants lead to the absorption of a non-absorbable drug, and the subsequent drug transportation routes through the membrane are discussed.
\end{abstract} 80

Keywords bioavailability improvement; suppository; latamoxef sodium; absorption promoter; diclofenac sodium; Tween

Generally, oral administration is the route of choice in the daily practice of pharmacotherapy. However, in some circumstances, this is impractical or even impossible, in the case of patients experiencing nausea and vomiting or convulsions, in operative patients, and before surgery. In these cases, the rectal route may represent a practical alternative; rectal administration is now a well accepted delivery route, for example, of anticonvulsants, nonnarcotic analgesics, theophylline, antiemetics, and antibacterial agents, and for inducing anesthesia in children. ${ }^{1)}$ Further, the rectal route has the advantage of allowing the drug to enter the systemic circulation without first passing through the liver.

We have shown that the nonsteroidal anti-inflammatory drugs (NSAID), diclofenac sodium(DF), phenylbutazone, and indomethacin (IM) enhanced rectal membrane permeability to non-absorbable drugs, such as sulfanilic acid (SA), creatinine, and insulin, and that the enhancing effects of the NSAID were not accompanied by irritation (histological change and release of protein from mucosa). ${ }^{2,3)}$ The absolute bioavailability of SA following administration in various suppository bases was sufficiently improved by the addition of DF alone. However, the rectal absorption of sulfaguanidine (SG) from lipophilic bases did not reach a sufficient level on the addition of DF only, ${ }^{4)}$ this difference being due to differences in the physicochemical properties of the drugs used.

When a drug is administered in suppository form, several factors may influence the extent and rate of drug absorption into venous blood. The drug released from the base must dissolve in the limited volume of rectal fluid (1 to $3 \mathrm{ml}),{ }^{5)}$ and the dissolved drug must then be taken up into the circulation through the relatively small surface of the rectum. The characteristics of the rectal preparation, e.g., whether it is liquid or solid, as well as the nature of the suppository base, appear to be important factors in determining the extent and rate of the release and absorption process of the drugs. ${ }^{6,7)}$

LMOX, an antibiotic, contains both a cationic and an anionic group in its molecule, and is administered clinically by injection because of its poor absorption via the gastrointestinal route. Since rectal administration is more convenient, and since the level of side effect is generally lower by this route, it is important to establish a method of rectal administration in which side effects due to adjuvants are minimal and in which the delivery system for non-absorbable drugs is safe and provides an effective blood level. Accordingly, in this study we examined the absorption of latamoxef sodium (LMOX) from suppositories containing adjuvants. We also examined the absorption route of the drug, through the lymphatic system from the rectum, in the presence of adjuvants.

\section{MATERIALS AND METHODS}

Materials LMOX(Shionogi Co., Ltd., Japan) and DF (Kodama Pharmaceutical Co., Ltd., Japan) were used as supplied. Witepsol H15 ${ }^{\circledR}$ (suppository base; Dynamit Novel Chemicals, Troisdrorf-Oberlat, West Germany) was purchased from Tokyo Kasei Kogyo Co. All other reagents and solvents were of reagent grade and were used without further purification.

Preparation of Suppository Finely-ground LMOX 
$(<74 \mathrm{~nm})$, prepared by sieving, was suspended in Witepsol H15 as a suppository base after the base had been melted. The molten mass was poured into suppository molds. The suppositories were cooled at $10^{\circ} \mathrm{C}$ and kept at $4{ }^{\circ} \mathrm{C}$ until use. Prior to use, the plastic molds were allowed to stand for $2 \mathrm{~h}$ at room temperature. Each suppository was conical in shape with a round apex, and measured $25 \mathrm{~mm}$ in length and $7 \mathrm{~mm}$ in maximum diameter, weighing approximately $1.0 \mathrm{~g}$. The drug content of the suppositories was determined to be within $\pm 0.3 \%$ of $50 \mathrm{mg}$.

In Vitro Release Study The in vitro release of LMOX from the suppositories at $37^{\circ} \mathrm{C}$ was determined with a dissolution-test apparatus for suppositories (Toyama Sangyo Co., Ltd.), carried out according to the method of Muranishi et al. ${ }^{8)}$ The released LMOX was determined by the spectrophotometric method.

Absorption Experiments Male Wistar rats, weighing $200-250 \mathrm{~g}$, were fasted for $24 \mathrm{~h}$ before the experiments, but water was given freely. The absorption experiments were carried out using anesthetized rats after the i.p. injection of Nembutal ${ }^{\circledR}$ sodium solution $(50 \mathrm{mg} / \mathrm{kg})$. After the insertion of a suppository into the rectum, the anus was closed with an adhesive (Aron $\mathrm{Alph}^{\circledR}$ ) to prevent leakage. This procedure resulted in no detectable leakage of rectal contents during the experiment. Blood samples were withdrawn from the jugular vein into heparinized syringes at designated intervals.

To measure the concentration of drug in the lymphatic fluid, the thoracic duct was cannulated with polyethylene tubing (PE 50) according to the method of Bollman, $\left.{ }^{9}\right)$ after an abdominal incision was made. Lymph fluid was sampled at 30-min intervals after rectal administration. The plasma samples and lymph fractions were taken into stoppered glass tubes and kept at $4{ }^{\circ} \mathrm{C}$ until assays were carried out.

Analytical Methods LMOX in saline for the in vitro release test was determined spectrophotometrically at $275 \mathrm{~nm}$ with a Shimadzu model UV-150-02 double beam spectrometer. LMOX levels in blood and lymph were measured by high pressure liquid chromatography (HPLC). Sample solutions were extracted with ethanol $(0.5 \mathrm{ml})$ and chloroform $(1 \mathrm{ml})$. The organic layer $(1 \mathrm{ml})$ was evaporated to dryness. The residue was redissolved with methanol and an aliquot was subjected to HPLC (Shimadzu LC-3A, Shimadzu, Japan) in a chromatograph equipped with a variable-wavelength ultraviolet detector (Shimadzu SPD-2) used in a reversed phase mode, the stationary phase being a Nucleosil ${ }_{5} \mathrm{Cl} 8$ column (Nargel Co., West Germany). A mixture of methanol-10 mM ammonium acetate buffer containing $50 \mathrm{~mm} n$-tert butyl ammonium bromide (1:2, by volume) was used as the mobile phase, and the flow rate was $0.8 \mathrm{ml} / \mathrm{min}$. The wavelength of the detector was set at $275 \mathrm{~nm}$.

Data Analysis The area under the blood concentration-time curves $(A U C)$ was calculated using the trapezoidal rule. All mean values are shown with the standard error (S.E.). The student's $t$-test was utilized to determine the significance of differences.

\section{RESULTS AND DISCUSSION}

The enhancing effects of DF on the rectal absorption of non-absorbable drugs have been studied by an in situ single perfusion method and by in vivo experiments. ${ }^{2,4)}$ DF enhanced rectal membrane permeability to nonabsorbable drugs. However, it was found that the absolute bioavailability of the drug depended on the physicochemical properties of the drug and the suppository base.

The rectal absorption of LMOX in rats after administration in the lipophilic suppository (Witepsol $\mathrm{H} 15)$ is shown in Fig. 1, in terms of the area under the blood concentration-time curves $\left(A U C_{0-2.5}\right)$ of $\mathrm{LMOX}$ for up to $2.5 \mathrm{~h}$. The $A U C_{0-2.5}$ of LMOX alone was low; on the addition of 10 or $50 \mathrm{mg} \mathrm{DF}$, the $A U C_{0-2.5}$ of LMOX had almost the same value as that of LMOX alone. DF did not significantly enhance the rectal absorption of LMOX, similarly to the case of SG in a lipophilic base, ${ }^{4}$ and the absolute bioavailability was in the range of $7 \%$ to $10 \%$ (with DF) compared with the bioavailability following i.v. injection. The enhancing effect of DF on SG absorption has also been observed in other lipophilic bases (Witepsol W35 and S55). ${ }^{4)}$ We concluded that the release of LMOX from the base was the rate limiting step in enhancing rectal absorption following administration of the suppository. Therefore, it is suggested that the release rate or dissolution rate to fluid in the rectal lumen may be an important factor in enhancing the absorption of LMOX following administration by suppository.

The rate and extent of rectal drug absorption is influenced by both the suppository base and the nature of the drug. The release rates of chloramphenicol, ${ }^{10}$ ) theophylline, ${ }^{11)}$ and paracetamol ${ }^{12)}$ from Witepsol H15 and H12 suppositories with added non-ionic surfactants, Tween 61 or Mirji 53, appeared to be higher than those of the drugs alone. The absorption data correlated with the in vitro release, suggesting that surfactants enhance drug absorption by increasing drug release from the base. The release rate of LMOX itself from the suppository base was relatively slow (Fig. 2). To increase this release rate, Tween 80 was added; this surfactant had no affect on the rectal membrane permeability to non-absorbable drugs or on the histological nature of rectal tissue at a concentration

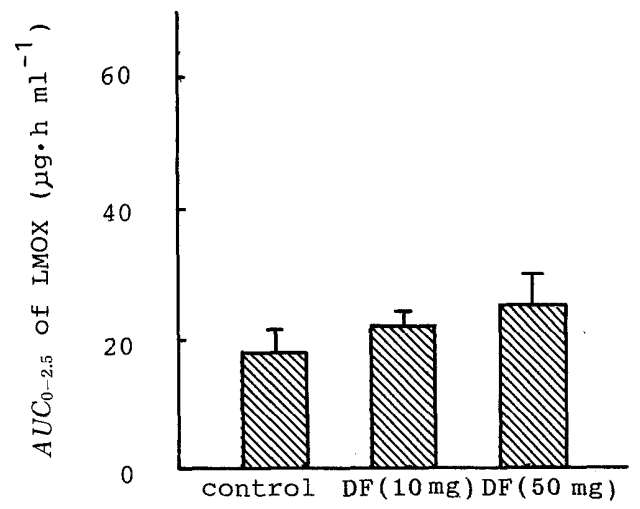

Fig. 1. Effects of DF on the Rectal Absorption of LMOX Following Administration by Suppository

Each column represents the mean values for 45 rats, with standard error shown as a bar. 


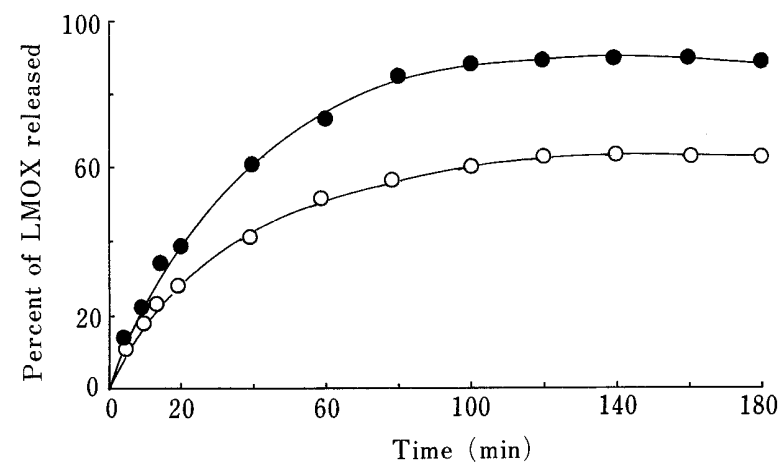

Fig. 2. Effects of Tween 80 on the Release Patterns of LMOX from Witepsol H15 Suppository in Saline

$$
\text { -O-, LMOX alone; - - } 1 \% \text { Tween } 80 .
$$

of $5 \% .^{13)}$ The release rate of LMOX from Witepsol $\mathrm{H} 15$ on the addition of Tween 80 at concentrations of $1 \%$ and $5 \%$ was significantly increased compared with the rate for LMOX alone. Forty percent of LMOX alone was released from $\mathrm{H} 15$ in $40 \mathrm{~min}$, while the same amount of the drug was released in $20 \mathrm{~min}$ from a suppository containing $1 \%$ Tween 80 . After $2 \mathrm{~h}$, the amount of LMOX alone released from the base was only $60 \%$, the release was not complete. However, on the addition of $1 \%$ Tween 80 , the release of LMOX was $94 \%$ in $2 \mathrm{~h}$, a significant improvement over the value for LMOX in base alone $(p<0.01)$. The release rate of LMOX with $5 \%$ Tween 80 was similar to that attained with $1 \%$ Tween 80 (no significance, data not shown). Thus, the release rate of LMOX from the lipophilic base was significantly improved by the addition of $1 \%$ Tween 80 .

To clarify whether the release pattern of LMOX was altered by the addition of DF, we also measured the release of LMOX on the addition of DF. The release rate of DF itself from a suppository is very fast, more than $90 \%$ of DF being released in $40 \mathrm{~min}$. However, DF did not influence the release of LMOX from the base. Therefore, the addition of DF was not a rate-limiting step in the release of LMOX from a base (data not shown).

The plasma level-time curves of LMOX (Fig. 3A) and the $A U C_{0-2.5}$ of LMOX ( Fig. 3B) on the addition of $5-50 \mathrm{mg}$ DF with or without $1 \%$ Tween 80 following suppository administration are shown in Fig. 3A, B. The rectal absorption of LMOX on the addition of DF $(50 \mathrm{mg})$ only, $1 \%$ Tween 80 only, or both $1 \%$ Tween 80 and DF $(5 \mathrm{mg})$ showed almost no increase compared with that of LMOX alone. However, when both DF $(10-50 \mathrm{mg})$ and $1 \%$ Tween 80 were used, the rectal absorption increased significantly, to about six times that achieved with the addition of DF $(50 \mathrm{mg}) ;(p<0.01)$. Figure $3 \mathrm{~A}$ showed that the maximum plasma level $\left(C_{\max }\right)$ of LMOX was obtained $20-30 \mathrm{~min}$ following the administration of suppositories containing both $1 \%$ Tween 80 and $10 \mathrm{mg}$ $\mathrm{DF}$; thereafter, the level slowly decreased. The $C_{\max }$ of LMOX was not altered within the range of 10 to $50 \mathrm{mg}$ of DF, however the elimination of LMOX was slower at a higher dose of DF. The plasma level of LMOX on the addition of $50 \mathrm{mg} \mathrm{DF}$ maintained $C_{\max }$ for a longer period than that achieved with $10 \mathrm{mg}$ DF. This may be due either to the continued absorption of LMOX from

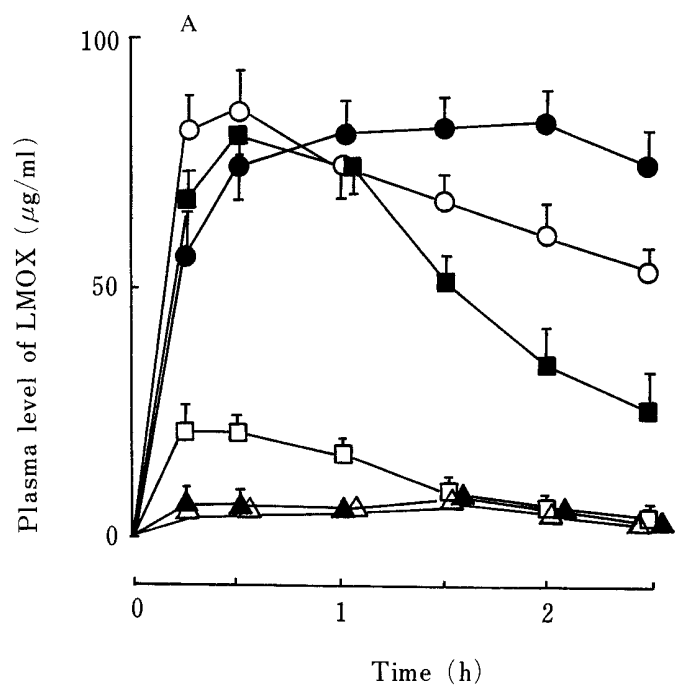

Fig. 3A. Effects of DF and Tween 80 on Plasma Level Time Curve of LMOX $(50 \mathrm{mg})$ Following Administration by Suppository

$-\triangle$ - , LMOX alone; - $\mathbf{\Delta - ,}$, LMOX and $1 \%$ Tween $80 ;-\square-$, LMOX, $1 \%$ Tween 80 and DF $(5 \mathrm{mg}) ;-$, LMOX, $1 \%$ Tween 80 and DF $(10 \mathrm{mg}) ;$ LMOX, $1 \%$ Tween 80 and DF $(20 \mathrm{mg})-1$ - LMOX, $1 \%$ Tween 80 and DF $(50 \mathrm{mg})$. Each point represents the mean values for $4-5$ rats with standard error shown as a bar.

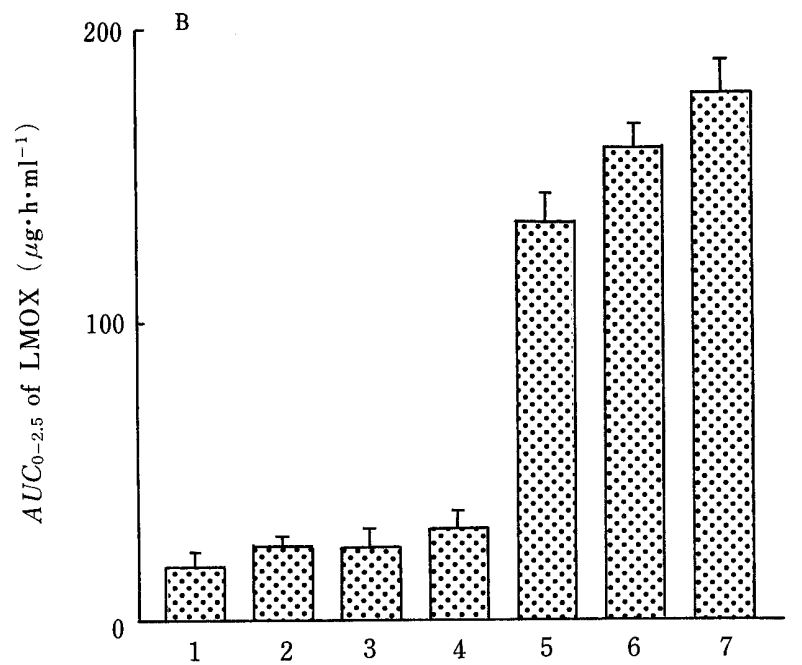

Fig. 3B. Effects of DF and Tween 80 on $A U C_{0-2.5}$ of LMOX (50 mg) Following Administration by Suppository

1, LMOX alone; 2, LMOX and DF (50 mg); 3, LMOX and 1\% Tween 80; 4, No. 3 and DF (5mg); 5 , No. 3 and DF $(10 \mathrm{mg}) ; 6$, No. 3 and DF $(20 \mathrm{mg}) ; 7$, No. 3 and DF $(50 \mathrm{mg})$. Each column represents the mean values for $4-5$ rats with standard error shown as a bar.

the rectum or to a reduction in the elimination rate from the kidney.

To confirm whether the elimination rate of LMOX was altered following administration in combination with $\mathrm{DF}$, we measured the elimination rate of LMOX after the i.v. administration of DF and LMOX together (Fig. 4). The pharmacokinetic parameters are summarized in Table I. The plasma level-time curves of LMOX following i.v. administration with and without DF were similar. There was no significant difference in pharmacokinetic parameters between LMOX alone and the combination of LMOX and DF. It has been shown that the elimination rate of LMOX following its administration alone and in combination with probenecid, an acidic drug, was similar, 
since LMOX appears to be excreted mainly through glomerular filtration. ${ }^{14)}$ Consequently, we assumed that DF in blood did not influence the elimination of LMOX via the kidney. With higher doses of DF, the prolonged $C_{\max }$ may be due to the continued absorption of LMOX from the rectum.

We have reported previously that DF and IM appeared to have a ceiling effect on apparent rectal clearance (blood to rectal lumen), the magnitude being dependent on the amounts of NSAID accumulated in the rectal tissue. ${ }^{2)}$ Therefore, it is possible that the plasma level of LMOX may depend on the dose of DF.

The apparent partition coefficients of LMOX to the organic solvents, chloroform and isoamyl alcohol, were small. It is possible that the apparent partition coefficient of LMOX may be altered from that of free LMOX, if a DF-LMOX complex is formed. However, we actually

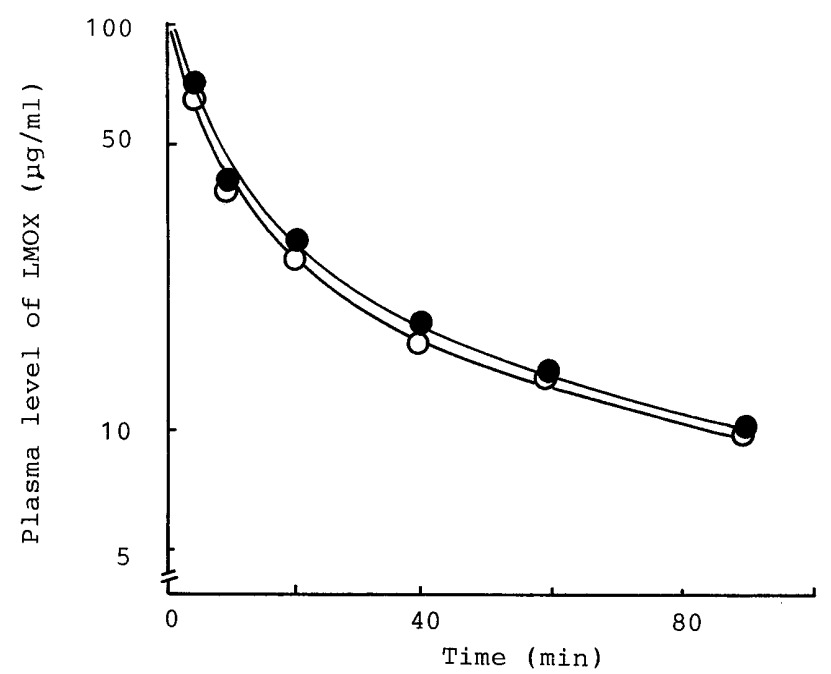

Fig. 4. Effects of DF on Plasma Level of LMOX Following Intravenous Administration

-1-, LMOX (10 mg) alone; -O-, LMOX (10 mg) and DF (5mg) combination. Each point represents the mean values for 3 rats. found that the values for the apparent partition coefficient of LMOX were the same in the presence or absence of DF (data not shown). Therefore, it appears that the prolonged absorption of LMOX in the presence of DF is due mainly to the interaction of DF with proteins and lipids in the rectal membrane. ${ }^{15)}$

The absolute bioavailability of LMOX following the rectal administration of the H15 suppository not including Tween 80 was only $7 \%$ of that achieved with i.v. administration. When Tween 80 and DF $(50 \mathrm{mg}$ ) were both present in the suppository, the absolute bioavailability increased remarkably, from $7 \%$ to $72 \%$; this preparation (1\% Tween 80 and $50 \mathrm{mg}$ DF combination) is thus very useful for rectal administration. $C_{\max }$ and $T_{\max }$ were not influenced by increasing amounts of DF, but an enhanced absolute bioavailability was obtained by prolonging the absorption process. The maintenance of higher plasma levels of LMOX may depend on the amount of DF added to the base.

It is well known that adjuvants significantly enhance both the blood level and the lymphatic uptake of both water-soluble and non-absorbable drugs. ${ }^{16)}$ Therefore, it

TABLE I. Pharmacokinetic Parameters of LMOX after i.v. Injection with and without DF

\begin{tabular}{|c|c|c|}
\hline & LMOX alone & with DF (5 mg) \\
\hline Dose $(\mu \mathrm{g})$ & 10000 & 10000 \\
\hline$C_{\mathrm{o}} \quad(\mu \mathrm{g} / \mathrm{ml})$ & $116.5(5.7)$ & $80.1 \quad(4.3)$ \\
\hline$V_{1} \quad(\mathrm{ml})$ & $85.8 \quad(10.3)$ & $123.8 \quad(11.3)$ \\
\hline$k_{12} \quad\left(\mathrm{~h}^{-1}\right)$ & $3.45(0.32)$ & $4.43(0.35)$ \\
\hline$k_{21} \quad\left(\mathrm{~h}^{-1}\right)$ & $2.77(0.29)$ & $3.69(0.39)$ \\
\hline$k_{\mathrm{e}} \quad\left(\mathrm{h}^{-1}\right)$ & $2.82(0.18)$ & $1.99(0.18)$ \\
\hline$A U C(\mu \mathrm{g} \cdot \mathrm{h} / \mathrm{ml})$ & $53.1 \quad(4.3)$ & $52.8 \quad(4.4)$ \\
\hline$C L \quad(\mathrm{ml} / \mathrm{h})$ & $188.3 \quad(15.1)$ & 189.4 (16.7) \\
\hline$V_{2} \quad(\mathrm{ml})$ & $107 \quad(11.6)$ & $150 \quad(18.5)$ \\
\hline$(\mathrm{ml})$ & $193 \quad(13.3)$ & (17.8) \\
\hline
\end{tabular}

These parameters were calculated from a two-compartment model. Each point represents the mean values $( \pm$ S.D. ) for 4 rats.

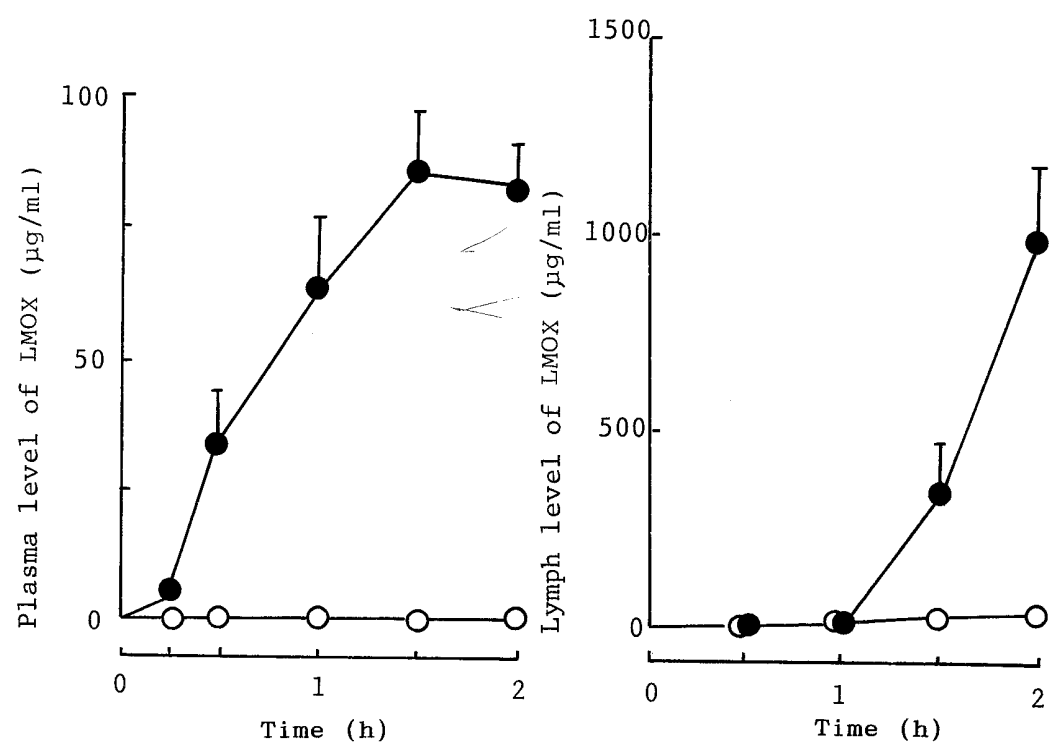

Fig. 5. Plasma and Lymph Levels of LMOX Following Rectal Administration by Suppository $-\mathrm{O}-$, LMOX $(50 \mathrm{mg})$ and $1 \%$ Tween $80 ;--$, LMOX $(50 \mathrm{mg}), 1 \%$ Tween 80 and DF $(50 \mathrm{mg})$. Each point represents the mean values for $4-5$ rats with
standard error shown as a bar. 
is important to study the contribution of the lymphatic system to more clearly understand the mechanism responsible for the enhanced rectal absorption of LMOX. Plasma and lymphatic LMOX levels were measured after the rectal administration of suppositories containing LMOX, 1\% Tween 80 and $50 \mathrm{mg}$ DF (Fig. 5). On the rectal administration of LMOX alone, there was no drug uptake into the lymphatic system. On the inclusion of Tween 80 and DF, the lymphatic level of LMOX was low in the early stage, but thereafter rapidly increasing with time. Similarly, plasma levels of LMOX were also sufficiently increased in this preparation. LMOX absorbed into the rectal membrane may be transported, via the blood stream and lymphatic flow, into systemic circulation. It has been demonstrated that the relatively large pore radius of the lymphatic capillary compared with the blood capillary in rats allows the lymphotropic delivery of compounds of high molecular weight (10 to $500 \mathrm{kDa}){ }^{6}$ ) However, it should be emphasized that the total amount of drug absorbed from the lymph system appears to be quite small, possibly because the lymph flow is low compared with the blood flow.

The combination of DF and Tween 80 enhanced the rectal absorption of the non-absorbable drug, LMOX, in both in vivo and in situ experiments. The rate and extent of LMOX released was sufficiently improved by the addition of Tween 80; this agent may decrease the interfacial tension between the drug and the dissolution medium. We found that the rectal absorption of the non-absorbable drug was achieved by improving the release rate from the base and by increasing the permeability of the rectal membrane to the drug.

The long term (more than 1 year) rectal use of some drugs in humans has been found to induce rectal ulceration, resulting in such clinical features as rectal bleeding and pain. ${ }^{17)}$ However, even the suppository base alone may compromise mucosal integrity, by causing epithelial cell loss and local inflammatory reactions. ${ }^{18)}$ If the damage is rapidly reversible, the occurrence of such mucosal damage does not necessarily preclude the clinical use of these bases. DF enhances rectal membrane permeability by binding with proteins and lipids in the rectal membrane, and this effect is reversed when the levels of DF in the rectal membrane are reduced. ${ }^{2)}$

The absorption of drugs through the intestinal and rectal membrane can occur via paracellular and transcellular routes, and DF has been found to enhance the permeability of the intestinal membrane to SA by both routes. ${ }^{19}$ LMOX, as well as SA, may also be transported via the paracellular and transcellular routes in the rectal membrane, and the lymphatic system also plays an important role in enhancing the absorption of LMOX following its administration together with adjuvants.

Further investigation is necessary to increase the safety of and to advance the development of a novel rectal delivery system for non-absorbable drugs.

\section{REFERENCES}

1) A. G. de Boer, L. G. J. de Leede, D. D. Breimer, Br. J. Anesth., 56, 69 (1984).

2) K. Nakanishi, A. Ogata, M. Masada, T. Nadai, Chem. Pharm. Bull., 32, 1956 (1984).

3) K. Nakanishi, M. Masada, T. Nadai, Chem. Pharm. Bull., 34, 2628 (1986).

4) K. Nakanishi, M. Masada, T. Nadai, J. Pharmacobio-Dyn., 13, 760 (1990).

5) A. G. de Boer, F. Moolenaar, L. G. J. de Leede, D. D. Breimer, Clin. Pharmacokinet, 7, 285 (1982).

6) T. Fukuda, Y. Takeuchi, Y. Hanyu, M. Yamashita, I. Kaneko, K. Tahira, Y. Fukumori, Yakuzaigaku, 40, 167 (1980).

7) T. Fujii, A. Kubota, N. Mizushima, Tokai J. Exp. Clin. Med., 7, 539 (1982).

8) S. Muranishi, Y. Okubo, H. Sezaki, Yakuzaigaku, 39, 1 (1979).

9) A. J. Bollman, J. C. Cain, J. H. Grindley, J. Lab. Clin. Med., 33, 1349 (1948).

10) A. A. Kassem, E. Nour El-Din, A. Abd El-Bary, H. M. Fadel, Pharmazie, 30, 472 (1975).

11) E. Minkov, N. Lambov, D. Kirchev, I. Bantutova, J. Tencheva, Pharmazie, 39, 242 (1984).

12) A. Abd Elbary, S. A. Ibrahim, H. Elsorady, H. A. Elmonem, Pharm. Ind., 45, 307 (1983).

13) K. Nakanishi, S. Miyazaki, M. Masada, T. Nadai, Yakugaku Zasshi, 102, 1133 (1982).

14) T. Yoshida, Y. Kimura, Y. Tochino, Chemotherapy, 28(S-7), 194 (1980).

15) K. Nakanishi, H. Saitoh, M. Masada, A. Tatematsu, T. Nadai, Chem. Pharm. Bull., 32, 3187 (1984).

16) T. Nishihata, K. Yasui, M. Yamazaki, A. Kamada, J. Pharmacobio-Dyn., 7, 278 (1984).

17) V. F. Eckardt, G. Kanzier, W. Remmele, Gastroenterology, 91, 1123 (1986).

18) E. J. Van Hoogdalem, C. Vermeij-Keers, A. G. de Boer, D. D. Breimer, J.Pharm.Sci., 79, 866 (1990).

19) S. Yamashita, H. Saitoh, K. Nakanishi, M. Masada, T. Nadai, T. Kimura, J. Pharm. Pharmacol., 39, 621 (1987). 